\title{
Schistosomose expérimentale
}

\author{
I. Étude de la fécondité de Schistosoma mansoni \\ en fonction de son adaptation \\ à la souche de Biomphalaria glabrata
}

\section{par Y.-J. GOLVAN, F. LANCASTRE, L. BARRIERE, F. DEROUIN et J. TRENEY-BOLOGNINI}

Laboratoire de Pathologie et Epidémiologie parasiaires ( $\mathrm{P}^{\mathrm{r}}$ Y.-J. GolvaN), Faculté de Médecine de Paris-Saint-Antoine, 27, rue de Chaligny, F 75571 Paris Cedex 12

\section{Résumé.}

L'infestation comparée de souches brésilienne (Recife), guadeloupéenne, martiniquaise et porto-ricaine de $B$. glabrata par 6 à 8 miracidiums de $S$. mansoni (Recife), a permis de faire les constatations suivantes:

$1^{\circ}$ Il existe une moindre résistance des Planorbes de Martinique et surtout de Guadeloupe ;

$2^{\circ}$ Les Planorbes guadeloupéens présentent le plus faible taux de positivité et le plus faible rendement moyen en cercaires, mais le rendement moyen par émission est assez comparable dans les 4 groupes;

$3^{\circ}$ De nombreux Planorbes - chez les groupes étudiés pendant une durée supérieure à deux mois - présentent des variations significatives périodiques dans leurs émissions de cercaires. Nous pensons que ces variations pourraient être provoquées par les maturations alternées de sporocystes issus d'un même miracidium ou de miracidiums différents.

$4^{\circ}$ L'infestation par S. mansoni agit fortement sur la fécondité des Planorbes, mais les œufs pondus se développent normalement.

\section{Summary.}

Experimental Schistosomiasis. I. Study of S. mansoni fecondity as regards to its adaptation to different Biomphalaria glabrata strains.

We compared the infestation of different strains of B. glabrata from Brasil (Recife), Guadeloupe, Martinique and Porto-Rico with 6 to 8 miracidia of $S$. mansoni (from Recife). We noted the four following points: 
$1^{\circ}$ The planorbid snails from Martinique and Guadeloupe had a low resistance to infestation.

$2^{\circ}$ The guadeloupean snails showed the lesser rate of positivity and the lower medium amount of emitted cercaries but, in the four strains of snails, the level of the issued cercaries is quite the same.

$3^{\circ}$ Many planorbid snails, in the groups studied during more than 2 months, showed significant periodic variations in the emission of cercaries. We thought that those variations might be caused by the alternate maturations of sporocysts born from the same miracidium or from different miracidia.

$4^{\circ}$ Infestation by $S$. mansoni had a strong effect on fecondity of the snails but the laid eggs had a normal development.

Les recherches entreprises depuis 1972 (1) dans notre laboratoire, sur l'écologie épidémiologique de la Schistosomose hépato-spléno-intestinale à Schistosoma mansoni en Guadeloupe, nous ont amenés à nous intéresser aux relations entre le parasite et son mollusque vecteur, le planorbe Biomphalaria glabrata, et en particulier à étudier la fécondité d'une souche de $S$. mansoni provenant de Recife (Brésil), au cours d'infestations expérimentales de quatre souches de Planorbes. De ces quatre souches, l'une provient également de Recife, les autres des îles antillaises de Martinique, Guadeloupe et Porto Rico (2).

De nombreux auteurs ont étudié les relations entre une souche de $S$. mansoni et diverses souches de Planorbes. Citons Files et Cram (1949), Files (1951), Kuntz (1952), Newton (1952), Barbosa et Barreto (1960), Paraense et Corréa (1963), Cridland (1970) et R. F. Sturrock et B. M. Sturrock (1970). A notre connaissance, seuls ces derniers et Files ont étudié l'infestation de plusieurs souches caraïbes en les comparant entre elles.

Entreprendre une expérimentation analogue s'est révélé indispensable pour la suite de nos travaux.

Deux points ont été particulièrement étudiés :

1) Le rendement de l'infestation d'une souche de Planorbes par la souche de Schistosome qui lui est naturellement adaptée. Le couple hôte-parasite de Recife que nous utilisons depuis une dizaine d'années a été choisi. Il constitue la référence « continentale» de base.

2) Le rendement de l'infestation des souches «insulaires» de B. glabrata par la souche brésilienne de $S$. mansoni.

(1) Ces travaux ont été possibles grâce au financement par la D.G.R.S.T., le C.N.R.S. et la C.N.A.M.T.S.

(2) Nous remercions très sincèrement $\mathrm{M}^{\mathrm{u}} \mathrm{A}$. Buttner (Institut de Parasitologie, Paris), $\mathrm{M}^{\mathrm{me}} \mathrm{J}$. Cénac (Laboratoire de Parasitologie de la Faculté de Pharmacie de Paris) et M. le Pr J. Lapierre (Laboratoire de Parasitologie de la Faculté de Médecine de Cochin Port-Royal, Paris), qui nous ont respectivement fourni les souches brésiliennes de $S$. mansoni et de $B$. glabrata (albinos), la souche de Porto Rico (albinos) et la souche de Martinique de B. glabrata. 


\section{Matériel et méthodes}

L'élevage des quatre souches de Planorbes s'effectue en chambre chaude, constamment éclairée, entre $22^{\circ}$ et $28^{\circ} \mathrm{C}$, dans des aquariums de 20 litres. Les mollusques sont nourris avec de la laitue fraîche. Les infestations se font individuellement par 6 à 10 miracidiums provenant d'œufs de $S$. mansoni récoltés dans le foie de Souris blanches, elles-mêmes infestées depuis 8 à 10 semaines par $200 \pm 50$ cercaires. Une fois infestés, les Planorbes sont gardés dans des bacs en verre de 5 litres.

A partir de la troisième semaine suivant la mise en contact avec les miracidiums, on cherche à provoquer l'émission de cercaires en plaçant chaque mollusque dans des tubes de Borrel contenant $5 \mathrm{ml}$ d'eau du robinet «vieillie » - pour la débarrasser de son chlore -, à $28-30^{\circ} \mathrm{C}$, sous fort éclairage, dans une enceinte à saturation d'humidité.

Après les tests, les mollusques sont gardés et nourris en récipient individuel en plastique de $200 \mathrm{ml}$.

Nous avons étudié, pendant quatorze semaines, chez les quatre groupes de Planorbes infestés :

$1^{\circ}$ La période prépatente, entre le jour de l'infestation et celui de la première émission cercarienne ;

$2^{\circ}$ La mortalité chez les Planorbes infestés;

$3^{\circ}$ L'évolution du nombre de mollusques exposés à l'infestation et émettant des cercaires ;

$4^{\circ}$ La quantité de cercaires émises dans chaque groupe et pour chaque mollusque positif ;

$5^{\circ}$ La fécondité chez les mollusques infestés.

\section{Résultats expérimentaux}

1) Pour les quatre souches de $B$. glabrata étudiées, la durée de la période prépatente a été de cinq semaines. Cette durée s'explique par la température modérée de l'eau des aquariums $\left(22^{\circ}\right.$ à $\left.26^{\circ} \mathrm{C}\right)$, et aussi par l'âge des Planorbes utilisés - 10 à 12 semaines, soit un diamètre de 6 à $12 \mathrm{~mm}$. Ces résultats sont conformes aux résultats trouvés au laboratoire par Stirewalt (1954) et par de nombreux autres auteurs (in Berrie, 1970).

2) Le pourcentage des Planorbes soumis à l'infestation et vivant à la cinquième semaine s'est montré variable selon les souches (tableau I et graphique 1), mais de façon non significative. 
Tableau I. - Pourcentage des Planorbes infestés survivants.

Souche

Brésil : 30 B. glabrata (albinos)

Guadeloupe : 18 B. glabrata

Martinique : $30 \mathrm{~B}$. glabrata

Porto Rico: 18 B. glabrata (albinos)

Cinquième
semaine

$93,3 \%$

$94,4 \%$

$83,3 \%$

$100 \%$
Quatorzième

semaine

$30 \%$

$5,5 \%$

$6,6 \%$

$11,1 \%$

Ces chiffres sont supérieurs à ceux donnés par Kuntz (1952), Barbosa et Barreto (1960), mais comparables à ceux obtenus par Cridland (1970) et Sturrock et Sturrock (1970).

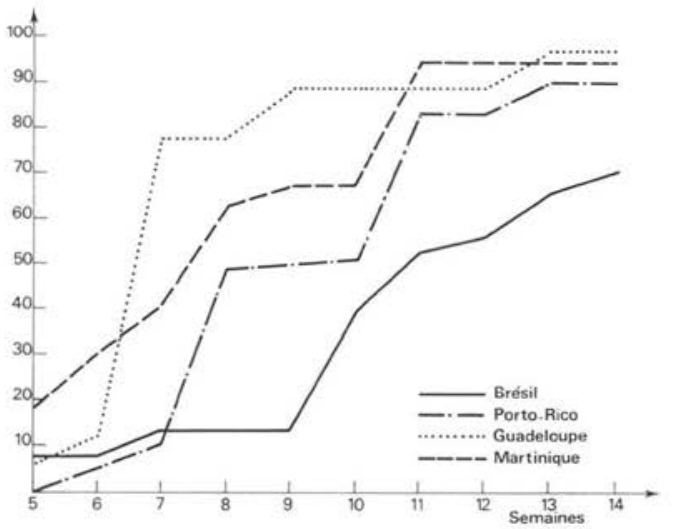

GraphiQue 1. - Mortalité chez les planorbes infestés.

Chez les groupes de Planorbes non infestés, la mortalité s'est révélée nettement inférieure, ce qui était prévisible. Il existe dans notre expérimentation une moindre résistance des Planorbes originaires de Guadeloupe et de Martinique, qui se manifeste dès les premières émissions cercariennes, et semble donc directement liée à l'infestation par la souche «étrangère » de Schistosomes (graphique 1). Les planorbes portoricains résistent mieux, et nous avons même $100 \%$ de survivants à la cinquième semaine de l'infestation, chiffre identique à celui trouvé par Sturrock et Sturrock (1970) (tableau II).

3) Le pourcentage des Planorbes qui émettent des cercaires varie entre la cinquième et la quatorzième semaine, en diminuant progressivement et de manière identique, peut-on dire, dans les quatres groupes étudiés (graphique 2).

C'est en effet à la première semaine d'émission (la cinquième de l'infestation) que nous trouvons les plus forts pourcentages de Planorbes positifs, et aussi presque tous ceux qui le seront jamais (graphique 3). Dès la septième semaine suivant l'infestation, il n'y a plus de mollusques guadeloupéens vivant. Chez les trois autres groupes, on constate un palier, voire une légère remontée entre les neuvième et dixième semaines, puis la décroissance se poursuit. A la quatorzième semaine, on ne trouve plus que des mollusques des souches brésilienne et martiniquaise qui soient encore vivants. 
TABleau II. - Pourcentages de Planorbes infestés survivants après 2 à 4 semaines d'après d'autres auteurs

\begin{tabular}{|c|c|c|c|c|}
\hline Auteurs & Année & $\begin{array}{c}\text { Origine et espèce des Planorbes } \\
\text { utilisés }\end{array}$ & $\begin{array}{l}\text { Souche } \\
\text { de } S \text {. mansoni }\end{array}$ & $\begin{array}{l}\text { Pourcen- } \\
\text { tages de } \\
\text { Planorbes } \\
\text { survivants }\end{array}$ \\
\hline Kuntz $\ldots \ldots \ldots$ & 1952 & $\begin{array}{l}\text { Venezuela (B. glabrata). } \\
\text { Porto Rico (B. glabrata). } \\
\text { Egypte (B. boissyi). }\end{array}$ & $\underset{\nexists}{\text { Egypte }}$ & $\begin{array}{l}68,8 \\
71,4 \\
69,5\end{array}$ \\
\hline Barbosa et Barreto & 1960 & $\begin{array}{l}\text { Bahia (B. glabrata). } \\
\text { Pernambuco (B. glabrata). }\end{array}$ & $\begin{array}{l}\text { Pernambuco } \\
\text { (Brésil) }\end{array}$ & $\begin{array}{l}52,9 \\
67,5\end{array}$ \\
\hline Cridland $\ldots . .$. & 1970 & $\begin{array}{l}\text { Soudan ( } B . \text { alexandrina alexan- } \\
\text { drina). } \\
\text { Egypte (B. a. alexandrina). }\end{array}$ & $\begin{array}{c}\text { Tanzanie } \\
\text {, }\end{array}$ & $\begin{array}{c}89,2 \\
60,8 \text { à } 97,6 *\end{array}$ \\
\hline $\begin{array}{l}\text { Sturrock et Stur- } \\
\text { rock } \ldots \ldots \ldots \ldots \ldots\end{array}$ & 1970 & $\begin{array}{l}\text { Porto Rico (B. glabrata). } \\
\text { Guyane }(B . \text { glabrata). } \\
\text { Dominique }(B . \text { glabrata). } \\
\text { R. Dominicaine }(\text { B. glabrata). } \\
\text { Trinidad }(B . \text { glabrata }) \text {. }\end{array}$ & 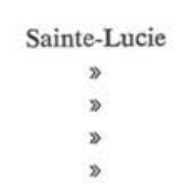 & $\begin{array}{l}100 \\
75,9 \\
96,6 \\
86,2 \\
78,1\end{array}$ \\
\hline
\end{tabular}

* Provenant de cinq colonies.

Nous donnons dans le tableau III les chiffres les plus significatifs concernant les Planorbes positifs.

Il apparaît donc que le taux de mortalité est variable chez les quatre groupes de Planorbes positifs, la différence étant causée essentiellement par le pourcentage initial de positifs, plus ou moins élevé suivant les souches. Là encore, les B. glabrata originaires de Guadeloupe sont exposés à une rapide disparition, étant donné leur faible taux initial de positivité, inférieur de

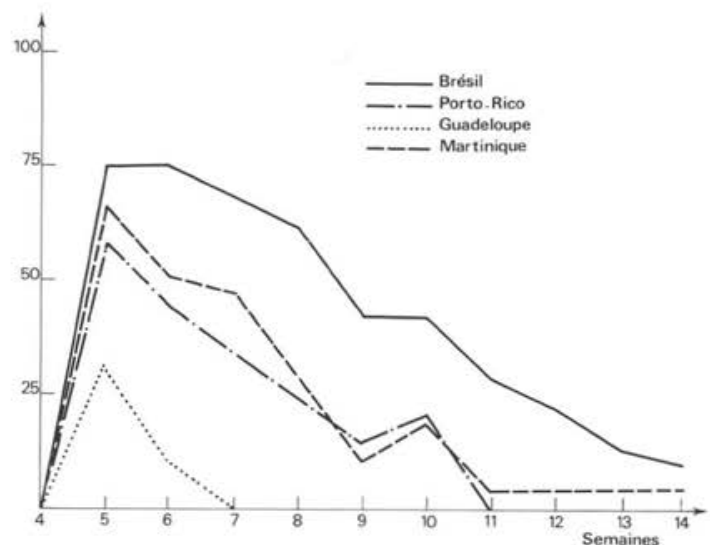

GRAPHiQue 2. - Pourcentage hebdomadaire de planorbes positifs. moitié à celui des autres souches antillaises et de deux fois et demie à celui de la souche brésiliene.

4) La production globale de cercaires est évidemment très importante chez les Planorbes du groupe brésilien, et c'est dans le groupe guadeloupéen qu'elle se montre 


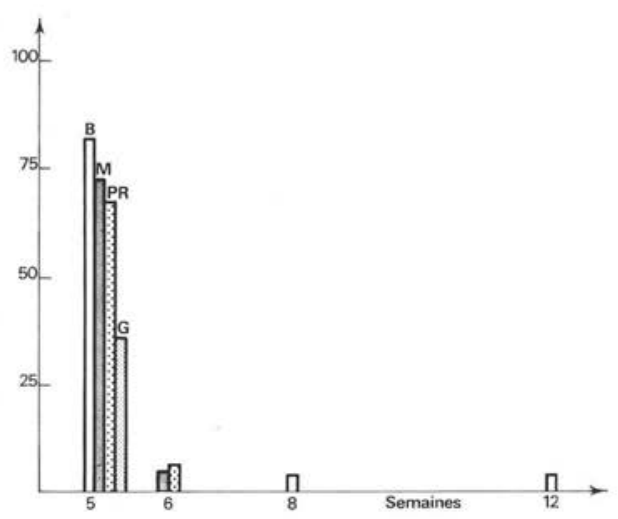

GraphiQue 3. - Pourcentage de planorbes devenant positifs.

la plus faible. La quantité de cercaires émises par mollusque positif est de 11070 par Planorbe brésilien, de 8520 par mollusque martiniquais, de 6370 par mollusque portoricain, mais seulement de 2330 par Planorbe guadeloupéen.

Le rendement de la souche guadeloupśenne est donc seulement de $21 \%$ par rapport à la souche de référence. Il est vrai que les émissions des mollusques guadeloupéens n'ont duré que deux semaines contre dix pour la souche continentale. Par ailleurs, si on étudie le rendement moyen de cercaires par émission, quel que soit le nombre de Planorbes positifs et la durée de l'infestation, on trouve des chiffres assez voisins (de 1750 à 2 490) pour les quatre souches. Et pour les deux premières semaines de positivité, les chiffres sont même de 1320 par émission de Planorbe brésilien, 985 par Planorbe portoricain et 1215 par Planorbe martiniquais, donc inférieurs aux 1750 cercaires par émission moyenne des mollusques guadeloupéens (tableau IV).

TABleau III. - Evolution de la positivité des Planorbes (en pourcentage par rapport au nombre de vivants à la cinquième semaine)

\section{Souches de B. glabrata}

Cinquième
semaine de
l'infestation

Brésil

Guadeloupe

Martiniqu

Porto Rico
82,1

35,3

72,0

66,6
Quatorzième semaine de l'infestation

10,7

0

4,0

0
Pourcentage maximum de mollusques positifs

89,3 (12 $2^{e}$ semaine)

35,3 (5॰ semaine)

76,0 (6 $6^{\mathrm{e}}$ semaine)

72,2 (6 $6^{\circ}$ semaine)

Tableau IV. - Production des cercaires dans les quatre groupes

$$
\begin{aligned}
& \text { Souches de } \\
& \text { B. glabrata }
\end{aligned} \begin{gathered}
\text { Production } \\
\text { totale de } \\
\text { cercaires }
\end{gathered}
$$
B. glabrata positifs

Nombre
d'émissions

Rendement par B. glabrata

$\begin{array}{rr}135 & 11070 \\ 8 & 2330 \\ 65 & 8520 \\ 40 & 6370\end{array}$

25
6
19
13

25
6
19
13

Moyenne des émissions

$$
\text { après après }
$$
7 semaines 14 semaines d'infestation d'infestation

$\begin{array}{rc}1320 & 2050 \\ 1750 & - \\ 1215 & 2490 \\ 985 & 2070\end{array}$




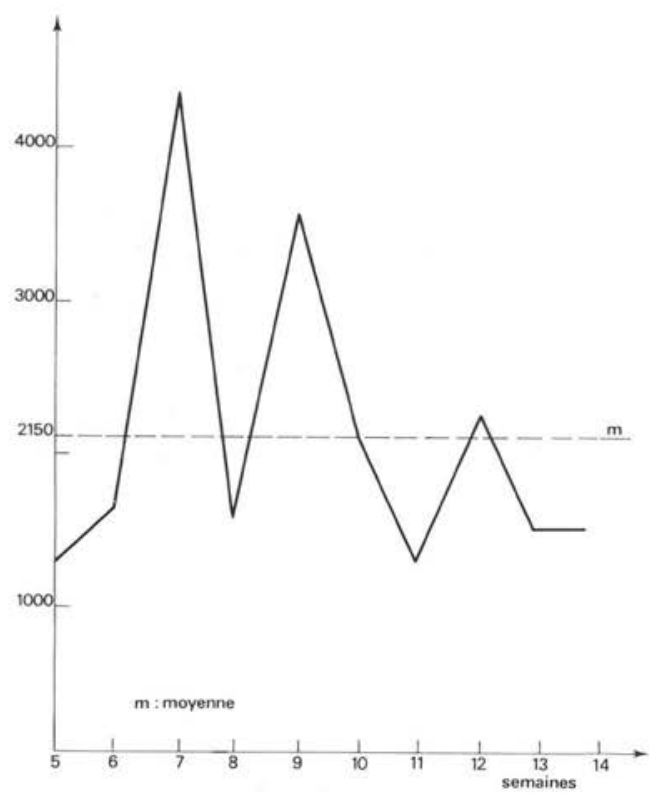

GrAPHIQUE 4. - Emissions hebdomadaires moyennes de trois planorbes brésiliens.

5) L'étude de la production hebdomadaire des Planorbes positifs n'a pu être menée à son terme que chez les mollusques brésiliens et martiniquais, seuls à avoir émis des cercaires jusqu'à la fin de l'expérimentation. Il s'agit des Planorbes brésiliens $B a, B h$ et $B a a$, et du Planorbe martiniquais $M w$.

Dans l'un et l'autre groupe, la production en cercaires des deux premières semaines est d'abord inférieure à la moyenne des dix semaines d'émission, puis une montée brutale se produit, suivie d'une chute, elle-même suivie d'une autre montée, etc. Cette alternance de forte et faible émission de cercaires est bien un phénomène commun aux deux souches de mollusques considérées (graphiques 4, 5 et 6).

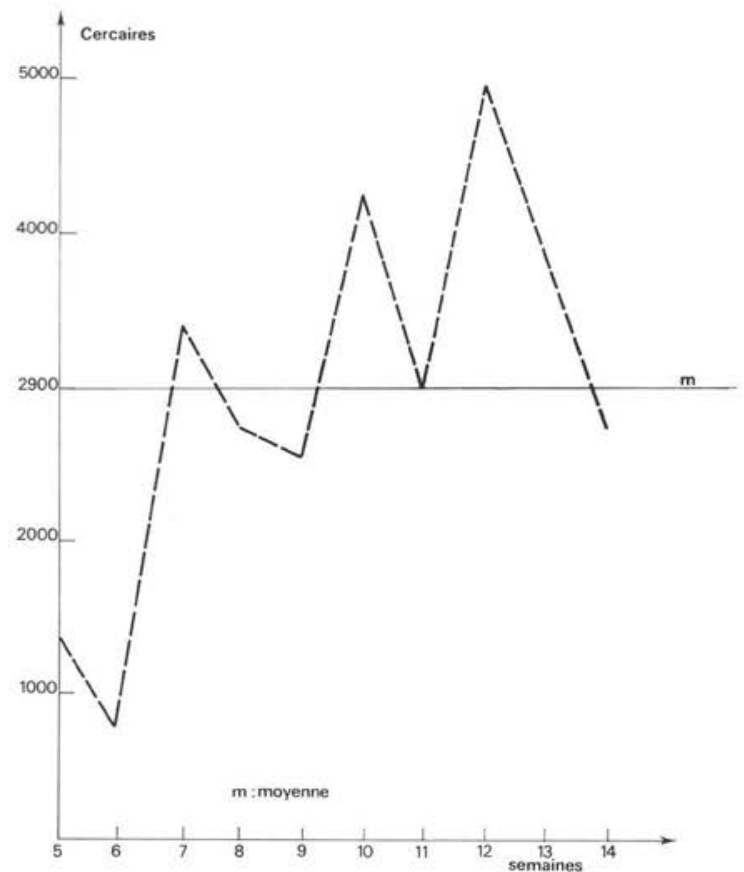

Graphique 5. - Emissions hebdomadaires d'un. planorbe martiniquais.

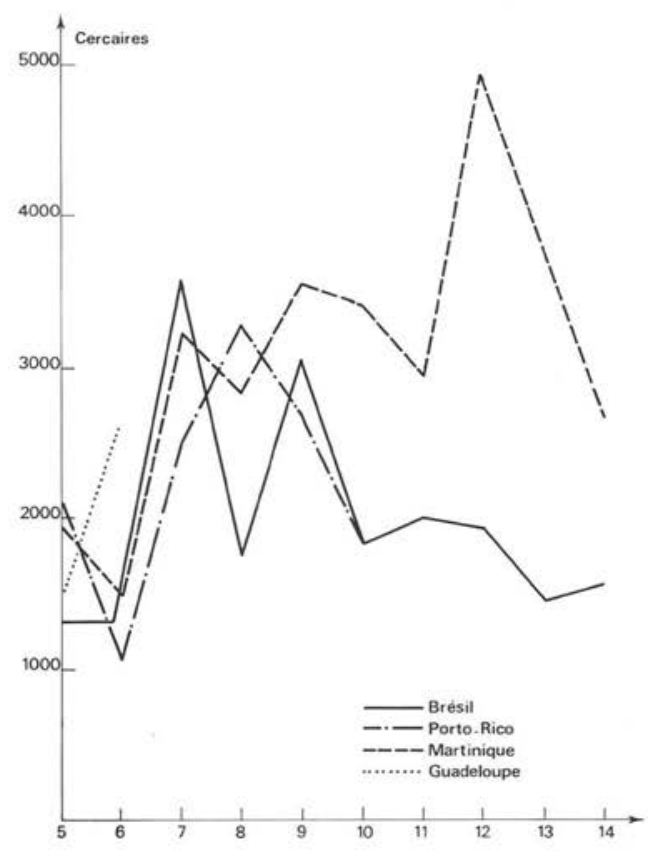

GRAPHIQUE 6. - Production hebdomadaire: moyenne de cercaires par planorbe positif. 
Déjà, en 1974, l'un d'entre nous avait observé un double pic dans l'émission de cercaires de $S$. mansoni par un groupe de Planorbes brésiliens testés chaque semaine (Mougeot et Golvan, 1974). Le phénomène avait alors été attribué à un développement inégalement rapide du parasite chez les mollusques, peut-être «en rapport avec une réaction de l'organisme du mollusque au parasitisme par $S$. mansoni »; les différents mollusques ne permettant pas la même vitesse de développement. Cet aspect de « double pic » dans la courbe d'émission des cercaires apparaissait comme un phénomène statistique, certains mollusques émettant à la huitième semaine, d'autres à la onzième. Mais la régularité et la nette amplitude de ces pics d'émission chez 10 des 24 Planorbes brésiliens positifs examinés par nous jusqu'au terme de nos observations sont en faveur d'un phénomène «normal» chez B. glabrata parasité par $S$. mansoni (tableau V). En effet, la date d'apparition du premier pic est la septième semaine dans 8 cas sur 10 ; le deuxième pic apparaît également 8 fois sur 10 à la neuvième semaine, et le troisième est évident à la douzième semaine chez trois des quatre Planorbes étudiés.

\section{Discussion}

Deux explications viennent aussitôt à l'esprit. On peut penser à une maturation successive ou cyclique des sporocystes issus d'un même miracidium et émettant leurs cercaires par vagues, qui vont s'amortissant ou s'exacerbant, suivant les souches de Planorbes. On peut aussi supposer que les sporocystes issus de plusieurs miracidiums suivent un développement alterné, la production maximum de chaque miracidium se signalant par un pic d'émission des cercaires.

Quoi qu'il en soit, cette succession assez régulière de pics n'est pas signalée dans les infestations réalisées par de très nombreux auteurs. Ceux-ci observent un pic après une période d'environ deux mois, puis une chute et le maintien de l'émission cercarienne à un niveau relativement constant jusqu'à sa fin (in Berrie, 1970).

L'amplitude décroissante des pics d'émission chez $B$. glabrata de Recife infesté par la souche de $S$. mansoni qui lui est adaptée peut s'expliquer par des phénomènes de résistance (immunitaire ?) qui se manifestent dans le cours de l'infestation et limitent à la longue la production de cercaires jusqu'à un niveau d'équilibre permettant une assez longue survie de l'hôte.

Dans le cas des Planorbes martiniquais, et en particulier du seul positif que nous avons pu étudier jusqu'au bout, la plus forte production de cercaires traduit peut-être un «emballement» de la production parasitaire, non contrôlée par le mollusque-hôte. Cette hypothèse n'est pas contradictoire avec la forte mortalité observée chez cette souche de Planorbes. Dans les autres groupes (portoricain et surtout guadeloupéen), la destruction de l'hôte proviendrait au contraire de violentes réactions de défense, également non compatibles avec la survie.

L'infestation par S. mansoni affecte manifestement la fécondité des Planorbes, quelle qu'en soit la souche. Nos relevés de pontes, qui ont débuté à la sixième semaine, soit une semaine après la première émission cercarienne, confirment ce fait. 


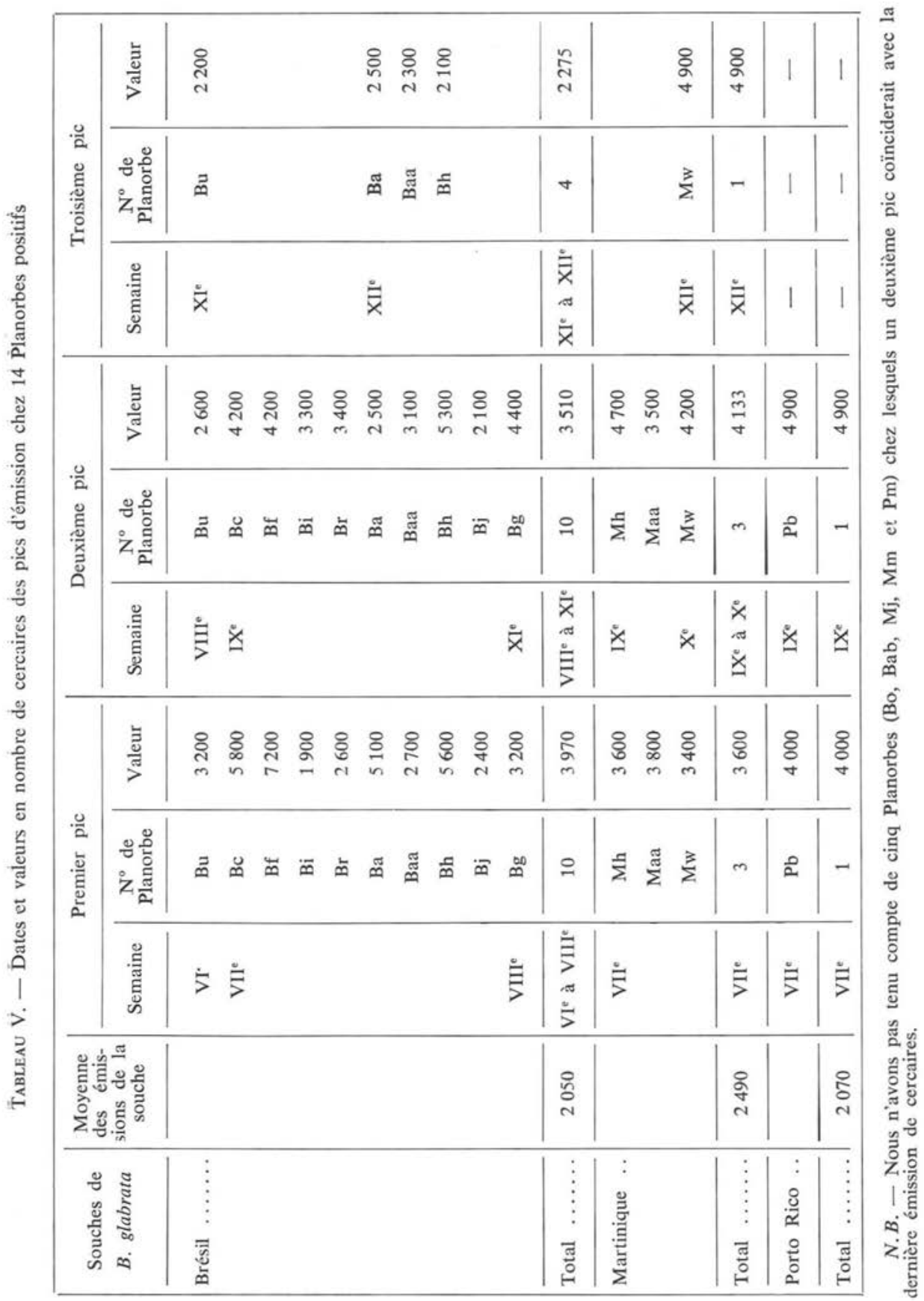


Les mollusques pondeurs étaient à cette date de :

- 10 B. glabrata de Recife sur 28 vivants, soit $35,7 \%$;

- 3 B. glabrata de Guadeloupe sur 16 , soit $18,7 \%$; à la septième semaine un quatrième Planorbe porte le pourcentage à $25 \%$;

- 3 B. glabrata de Martinique sur 21 , soit $14,3 \%$;

- 3 B. glabrata de Porto Rico sur 17 , soit $17,6 \%$.

A titre de comparaison, le pourcentage de pondeurs chez des Planorbes non infestés et placés dans les mêmes conditions peut atteindre $60 \%, 80 \%$ et même plus. Un travail est en cours pour préciser l'ampleur de cette réduction de la fécondité chez la souche brésilienne. Cependant, nous n'avons pas observé de différences significatives dans le nombre moyen de pontes et d'œufs, entre les Planorbes positifs et les négatifs (tableau VI).

Signalons aussi que nous n'avons pas constaté de différence dans le développement des œufs pondus par les Planorbes infestés comparés à des Planorbes sains, la rapidité de ce développement dans chaque souche étant seulement fonction de la température de l'eau.

Tableau VI. - Fécondité des Planorbes positifs et négatifs entre la $6^{e}$ et la $14^{e}$ semaine de l'infestation.

\begin{tabular}{|c|c|c|c|c|c|c|c|c|}
\hline \multirow{2}{*}{ Souches de $B$. glabrata } & \multicolumn{4}{|c|}{ Planorbes pondeurs } & \multicolumn{4}{|c|}{$\begin{array}{c}\text { Moyenne des pontes } \\
\text { et cufs hebdomadaires } \\
\text { par Planorbe }\end{array}$} \\
\hline & $\begin{array}{c}\text { Nom- } \\
\text { bre }\end{array}$ & $\%$ & $\begin{array}{c}\text { tifs } \\
\text { Posi- }\end{array}$ & $\begin{array}{l}\text { Néga- } \\
\text { tifs }\end{array}$ & $\begin{array}{l}\text { chez } \\
\text { Pontes }\end{array}$ & $\begin{array}{l}\text { ositifs } \\
\text { Cufs }\end{array}$ & $\begin{array}{c}\text { chez } \\
\text { Pontes }\end{array}$ & $\begin{array}{c}\text { négatifs } \\
\text { CEufs }\end{array}$ \\
\hline Brésil $\ldots \ldots \ldots \ldots \ldots \ldots$ & 10 & 35,7 & 7 & 3 & 4,8 & 11,3 & 5,1 & 12,6 \\
\hline Guadeloups $\ldots \ldots \ldots \ldots$ & 4 & 25,0 & 0 & 4 & 0 & 0 & 3,3 & 10,6 \\
\hline Martinique $\ldots \ldots \ldots \ldots$ & 3 & 14,3 & 1 & 2 & 2,5 & 10,6 & 3,0 & 12,8 \\
\hline Porto Rico $\ldots \ldots \ldots \ldots$ & 3 & 17,6 & 0 & 3 & 0 & 0 & 4,4 & 10,2 \\
\hline
\end{tabular}

\section{Conclusion}

Il ressort de ce travail que le comportement de la souche brésilienne de $S$. mansoni est différent selon que celle-ci se développe chez la souche de B. glabrata à laquelle elle est adaptée de longue date, ou chez les Planorbes provenant des Antilles. De ceux-ci, ce sont les mollusques provenant de Guadeloupe qui paraissent les moins bien adaptés à la souche brésilienne de Schistosome. Ceux de Porto Rico et de Martinique occupent une situation intermédiaire. En effet, si les pourcentages de mortalité après cinq semaines d'infestation sont, en gros, très comparables, on observe qu'ils sont bien plus importants à la quatorzième semaine dans le groupe antillais. De même, il existe des différences remarquables dans les pourcentages de mollusques émettant des cer- 
caires — cela dès la première semaine d'émission - et, bien entendu, dans le rendement brut.

Mais il existe des similitudes. Tout d'abord, la durée de la période prépatente de l'infestation est la même dans les quatre groupes; ensuite, les moyennes hebdomadaires d'émission sont du même ordre de grandeur, et surtout analysés chez les individus des souches brésilienne et martiniquaise qui ont produit des cercaires jusqu'au terme de l'expérimentation, ces émissions varient presque simultanément et de façon périodique. C'est à notre connaissance la première relation d'un tel phénomène dans les infestations de B. glabrata par $S$. mansoni.

Nous pensons qu'il est dû au développement alterné de sporocystes issus d'un même miracidium ou même de miracidiums différents. Des travaux sont en cours pour apprécier la constance de ce phénomène et son influence sur le mollusque-hôte, et en particulier sur la fécondité de ce dernier, qu'il appartienne à une souche déterminée ou provienne d'une hybridation.

\section{Bibliographie}

Barbosa (F.S.) et Barreto (A.C.), 1960. - Differences in susceptibility of Brasilian strains of Australorbis glabratus to Schistosoma mansoni. Expl. Parasit., 9, 137 140.

BerRIE (A. D.), 1970. - Snails problems in African Schistosomiasis. Advances in parasitology, vol. 8, Academic Press, Publ., London and New-York, 313 p.

CRIDland (C. C.), 1970. - Susceptibility of the snail Biomphalaria alexandrina alexandrina from the UAR and the Sudan to infection with a strain of Schistosoma mansoni from Tanzania. Bull. Org. Mond. Santé (Bull. Wld. Hlth. Org.), 43, 809-815.

Files (V.S.), 1951. - A study of vector-parasite relationships in Schistosoma mansoni. Parasitol., 41, 264-269.

Files (V.S.) et Cram (E. B.), 1949. - A study on the comparative susceptibility of snails vectors to strains of Schistosoma mansoni. J. Parasit., 35, 555-560.

KUNTZ (R. E.), 1952. - Exposure of planorbid snails from the western hemisphere to miracidia of the Egyptian strain of Schistosoma mansoni. Proc. Helm. Soc. Wash., 19, 9-15.

Mougeot (G.) et Golvan (Y.-J.), 1974. - Etude des relations entre la longévité de Biomphalaria glabrata et son infestation par Schistosoma mansoni (Etude de la variation quantitative de l'émission des furcocercaires). Bull. Soc. Sci. Vet. Med. comparée Lyon, 76, 119-124.

Newton (W. L.), 1952. - The comparative tissue reaction of two strains of Australorbis glabratus to infection with Schistosoma mansoni. J. Parasit., 38, 362-366.

Paraense (W. L.) et Correa (L. R.), 1963. - Variation in susceptibility of populations of Australorbis glabratus to a strain of Schistosoma mansoni. Rev. Inst. Med. trop. São Paulo, 5, 15-22. 
Stirewalt (M. A.), 1954. - Effect of snail maintenance temperatures on development of Schistosoma mansoni. Expl. Parasit., 3, 504-516.

StURrock (R.F.) et StURrock (B. M.), 1970. - Observations on the susceptibility to Schistosoma mansoni from St. Lucia of several caribbean strains of snails of the genus Biomphalaria. W. Ind. Med. J., 19, 9-13. 